\title{
Simulation numérique et conception des structures de grands navires
}

\author{
FRANÇOIS BESNIER ${ }^{\mathrm{a}}$ \\ PRINCIPIA MARINE, 1 rue de la Noë, BP 2212, 44321 Nantes Cedex 3, France
}

Reçu le 14 janvier 2005, accepté le 13 octobre 2005

\begin{abstract}
Résumé - La simulation numérique joue un rôle croissant dans la conception des navires à côté des ou associée aux approches réglementaires édictées par les sociétés de classification. Ce texte, non exhaustif, veut illustrer les tendances actuelles et les difficultés rencontrées dans l'approche simulation numérique. Celles-ci sont de plusieurs ordres : problèmes de modélisation liés à la taille des structures et à la nécessité d'une approche multi-échelle, problèmes de méthodologie liés à la complexité de l'environnement, problèmes de couplage fluides structures. Enfin ces études doivent se dérouler dans un temps extrêmement court, avec des informations qui se précisent au cours du déroulement de l'étude, informations entachées d'incertitudes et de dispersions. Les grands codes éléments finis généralistes disponibles sur le marché sont en général utilisés, associés à des outils et développements spécifiques si nécessaire, dans des chaînes de calcul plus ou moins intégrées. Les situations extrêmes ou accidentelles font l'objet d'études assez sophistiquées, nécessitant un travail d'interprétation important.
\end{abstract}

Mots clés : Calcul de structure / fatigue / fiabilité / simulation numérique / dimensionnement

\begin{abstract}
Numerical simulation plays an increasing role in ship design, besides or associated to rule based approach, based on Classification Societies rules. This non exhaustive paper aims at illustrating tendencies and difficulties encountered in the numerical simulation usage in maritime industry. These are of different kind: modelling problems associated to the structures size, need of multi-scale approach, methodology issues associated to the complexity of the ship environment, coupled fluid structure problems. Last but not least, theses studies must be achieved in a very short delay with information rapidly changing during the project, information marred with uncertainties sand dispersion. Large commercial software available on the market are generally used, but associated to specific extensions. Complex studies are performed in the simulation of extreme or accidental situation, with the need of an important result interpretation.
\end{abstract}

Key words: Structural analysis / fatigue / reliability / numerical simulation / dimensioning

\section{Introduction}

La construction navale est une activité humaine très ancienne et l'on retrouve de nombreux ouvrages, archives et documents et ce, depuis l'antiquité, traitant de la conception des navires. Citons plus récemment la construction des navires marchands, cours professé par Henri Charpentier à l'école d'application du Génie maritime, édité par Dunod en 1936, qui donne une bonne idée de l'état de l'art avant l'apparition des outils et méthodes modernes de calcul. C'est un domaine qui comme beaucoup d'autres, a vu ses méthodes de conception évoluer avec le développement des ordinateurs et méthodes de

\footnotetext{
a Auteur correspondant :

francois.besnier@principia.fr
}

calcul numérique (Masson [1]). Cependant, un certain nombre de spécificités ont freiné la généralisation de la simulation numérique en construction navale.

Mentionnons tout d'abord la difficulté de modélisation de l'environnement : une structure souple se déplaçant sur la surface libre séparant deux fluides, surface libre très changeante et complexe dans le temps et dans l'espace. D'autre part, les profils de missions et donc les conditions de mer que le navire pourra rencontrer au cours de ses 20 à 40 ans de vie sont susceptibles d'évoluer en fonction des différents armateurs et ne peuvent donc qu'être estimées en probabilité. Mentionnons enfin la possibilité d'évolutions climatiques sur une aussi longue durée, ce qui complique encore le problème.

Le deuxième élément spécifique est lié à la structuration de la profession et à la place importante des 
sociétés de classification (ex. Bureau Veritas [2]). En effet, la conception des structures s'appuie essentiellement sur les règlements édités par les Sociétés de Classification, chargées d'approuver les plans des navires. Ces règlements reposent sur le retour d'expérience acquis et mis à jour par la surveillance d'un très grand nombre de navires. La réglementation est a priori conservative et prudente. Mais cette situation a tendance à évoluer et les Sociétés de Classification admettent des conceptions justifiées par simulation numérique. Il est clair que dans le cas de nouveaux concepts ou de structures innovantes, il n'existe pas de retour d'expérience et la simulation numérique trouve alors toute sa place. Par ailleurs, la compétition mondiale pousse à optimiser les structures ce qui implique de mieux connaître les marges, donc justifie des analyses plus fines. Enfin, il existe des domaines non couverts par la classe comme les vibrations et le confort, les explosions et collisions qui sont abordés par le calcul numérique. Pour compléter ce tour d'horizon, ajoutons que la conception des navires militaires s'effectue dans un contexte différent et que la simulation y occupe une place importante.

On s'oriente donc de plus en plus, comme dans d'autres industries, vers le concept de maquette numérique et la conception rationnelle en ce qui concerne la structure ( first principle ou rational design »). Dans le domaine de l'hydrodynamique et de la tenue à la mer, les essais en bassin effectués sur des maquettes à échelle réduite restent une étape obligée et incontournable, malgré les progrès remarquables des codes d'hydrodynamique.

\section{Contexte industriel et spécificités des navires}

Par la suite, on s'intéressera essentiellement aux grands navires bien que certaines remarques puissent s'appliquer également à tout type de navire. Les grands navires tels que ceux fabriqués par les grands chantiers européens, comme les navires de croisière, les méthaniers, sont en général construits à l'unité ou en très petites séries. Il n'y a pas de prototype et chaque navire est un nouveau projet. Ces navires sont conçus et construits dans des délais extrêmement courts au regard de leur complexité, typiquement de 24 à 32 mois de la signature à la livraison pour un grand paquebot. Or la phase avant projet/projet qui précède la signature ne conduit qu'à la définition des grandes dimensions, un premier dessin, un premier devis de poids (avant-projet) précisé au cours de la phase projet par la définition des grandes surfaces, le type de raidissage. La phase de conception ne commence qu'après la signature, reprenant les travaux issus de la phase projet et réalisant au Bureau d'Études les dessins détaillés qui devront être soumis pour approbation aux Sociétés de Classification. Cette phase de conception détaillée est très courte, de l'ordre de six mois pour un grand navire.

Les grands navires sont des structures réalisées en tôles d'acier laminées, les matériaux utilisés étant l'acier E24, l'acier à haute limite élastique E36 ou des alliages légers dans les parties hautes. Ces tôles sont assemblées entre elles par soudage à l'arc, avec électrode permanente et apport de fil sous gaz inerte (TIG) ou électrode consommable (MIG), ce qui crée des déformées et contraintes en particulier pour les tôles les plus minces. Ces déformations doivent être réduites par chauffage et posent des problèmes à l'assemblage des différents blocs qui constituent le navire (Chau et al. [3]). Les techniques de soudage laser, qui ne créent pas d'effets induits, ont maintenant dépassé le stade expérimental et plusieurs chantiers comme Meyerwerft et AKER en Allemagne, Fincantieri en Italie ont investi dans des bancs de soudage laser pour les panneaux plans et mettent de plus en plus largement en ouvre cette technique dont l'utilisation devrait se développer dans le futur. Les navires rapides, catamaran ou monocoques sont souvent réalisés en aluminium pour des longueurs inférieures à $100 \mathrm{~m}$. Les épaisseurs varient de $20 \mathrm{~mm}$ dans les fonds à $4 \mathrm{~mm}$ dans les ponts supérieurs.

L'organisation des grandes surfaces du navire, bordés de carène, ponts et cloisons longitudinales permet de reprendre les efforts de flexion, torsion et cisaillement du navire considéré comme une poutre. Un deuxième niveau de raidissage est constitué par les couples renforcés tels que les cloisons transversales et les anneaux porques (Fig. 1).

Un troisième niveau est celui des ponts et panneaux raidis. On a donc des structures comportant des ensembles quasi-périodiques de tôles raidies, s'appuyant sur une ossature orthogonale, avec une hiérarchie de raidissage primaire, secondaire et tertiaire, les raidisseurs contribuant par leur section à augmenter le module d'inertie de la poutre navire et jouant par ailleurs un rôle antiflambement important.

Les navires classiques se présentent donc comme des structures élancées dont l'étude, en première approximation peut se faire à partir d'un modèle poutre de Timoshenko, avec prise en compte des déformations dues aux efforts tranchants. Ce type de modèle est bien adapté à des navires de charge ou des pétroliers. Il est déjà plus discutable pour des navires à ponts ouverts comme les porte-conteneurs et ne fournit qu'une grossière approximation dans le cas des paquebots, dont la structure longitudinale est interrompue par de grandes ouvertures tout comme les bordés, ceux-ci au-dessus du pont à embarcations, dit pont résistant. Les armateurs recherchent en particulier des paquebots offrant un maximum de cabines avec balcons et vue sur la mer ce qui a tendance à multiplier les ouvertures dans le bordé.

\section{L'approche traditionnelle de la conception}

Les spécifications générales d'un navire pour une mission et un programme définis, tiennent en peu de chiffres : longueur, largeur, déplacement, tirant d'eau, tirant d'air, vitesse contractuelle. Dans le cas d'un paquebot, le nombre de cabines, de ponts est également précisé. L'architecte naval du projet définit le plan d'ensemble (General Arrangement) et l'échantillonnage de la coupe 


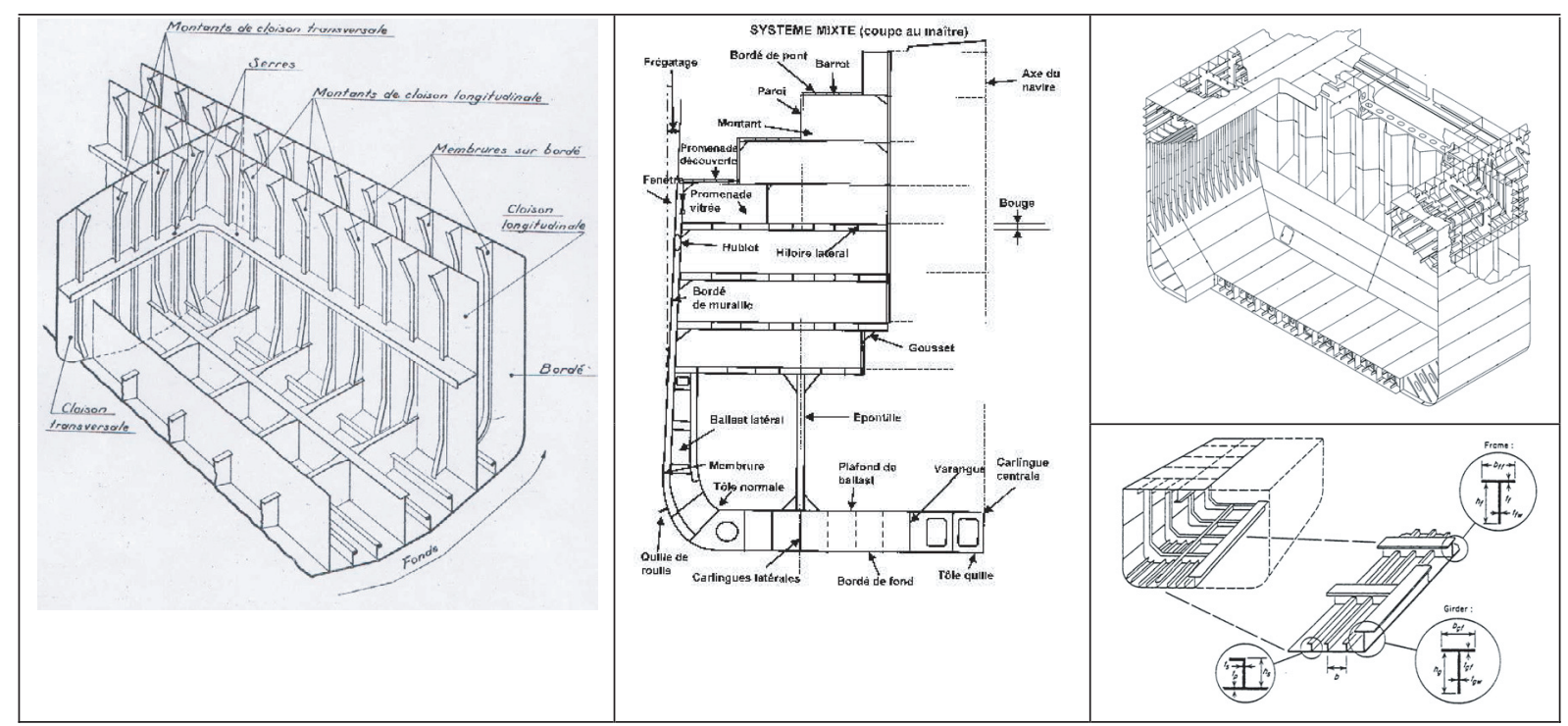

Fig. 1. Organisation structurelle.

au maître. Cet échantillonnage doit permettre au navire, considéré comme une poutre, de résister au moment dimensionnant tel que fourni par la classe et correspondant à un chargement dimensionnant majorant. À ce niveau (avant-projet, projet), les calculs réalisés sont assez simples et s'appuient sur des formules semi-analytiques ou des modèles poutres et sur les formules réglementaires des Sociétés de Classification, intégrées de plus en plus dans des logiciels simples.

\subsection{Calculs réglementaires}

À partir de la signature de la commande, le Bureau d'Études précise le dimensionnement s'appuyant sur des modèles éléments finis, sous un certain nombre de cas de charge réglementaires : navire en eau calme, roulis, en arc et contre-arc, ces deux derniers cas correspondant au navire sur houle réglementaire. Notons que ces calculs doivent être effectués en condition libre-libre sous chargements équilibrés. On utilise en général la méthode d'équilibrage inertiel telle que décrite par exemple par Gérardin et Rixen [4]. Il est également intéressant de noter que ces calculs sont des calculs statiques ou quasistatiques, bien qu'à l'évidence le comportement du navire soit un comportement dynamique. Dans ces modèles éléments finis, on utilise un pas de maillage correspondant au raidissage primaire. Les raidisseurs secondaires sont pris en compte soit en reportant leurs sections et inerties sur les lignes de raidisseurs voisines, soit en utilisant des éléments orthotropes. Enfin, en ce qui concerne les zones d'ouvertures non modélisées explicitement, des méthodes d'homogénéisation permettent de définir des propriétés équivalentes. Les contraintes obtenues par ces calculs globaux sont comparées à des valeurs réglementaires, ce qui fournit un premier niveau de validation de l'échantillonnage (Fig. 2).

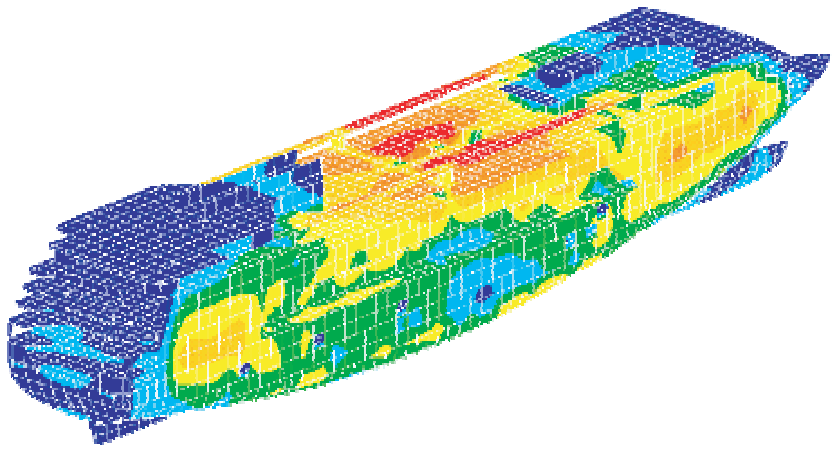

Fig. 2. Contraintes obtenues sur le modèle global.

Les résultats de ces calculs sont ensuite utilisés pour charger des modèles locaux beaucoup plus détaillés, dont les résultats sont confrontés aux valeurs réglementaires couvrant la tenue en fatigue et le flambement (Fig. 3). À ce niveau, des itérations portant sur l'échantillonnage (augmentation locale des épaisseurs en introduisant des « inserts »), le choix des matériaux (passage en acier à haute limite élastique), la modification de la topologie et/ou de la géométrie sont généralement effectuées afin de satisfaire aux critères des sociétés de classification.

Cette démarche a fait ses preuves et est adaptée au contexte particulier de la construction navale à l'unité. Le devis de masse en particulier, donc le chargement évolue au cours de la conception. Les contraintes d'emménagement peuvent amener à modifier la géométrie ou la topologie du navire. D'une façon générale, au cours des six mois environ consacrés à la conception au BE, les informations se précisent de plus en plus, imposant des boucles très rapides de validation.

On peut cependant déjà apporter un certain nombre de critiques à cette démarche.

La première tient au transfert des chargements du modèle global au modèle local. Faut-il charger en 


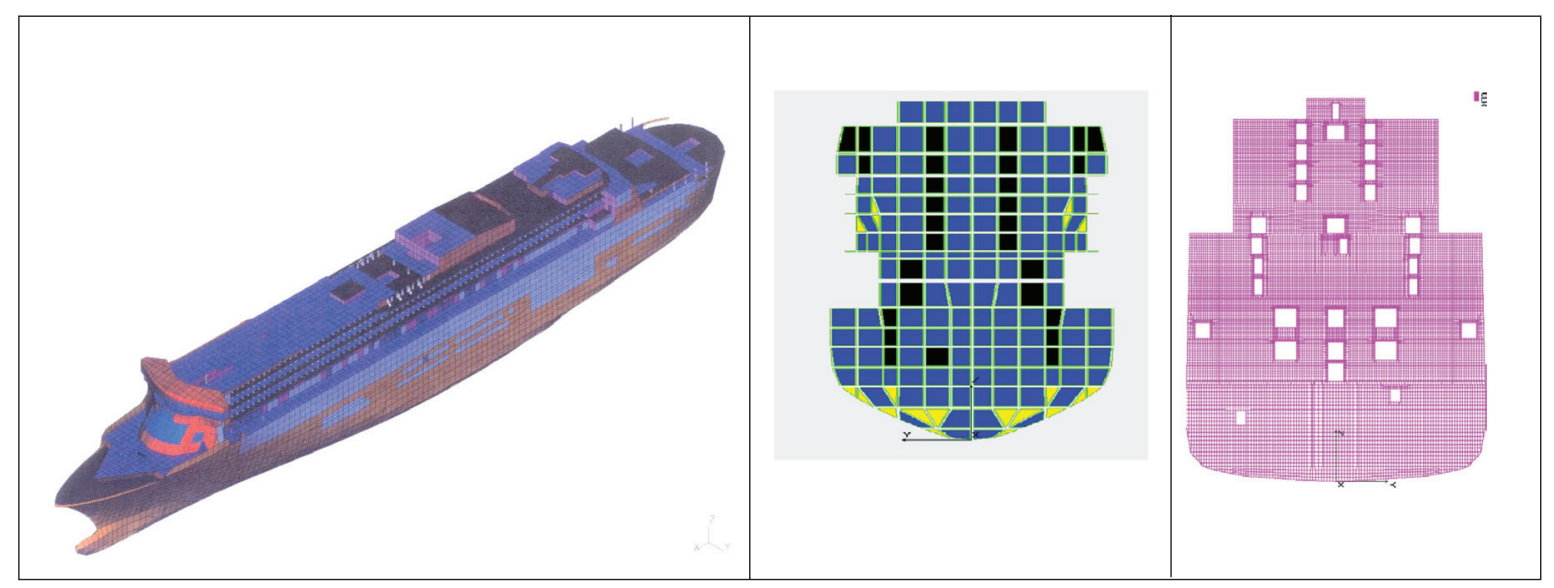

Fig. 3. Du modèle global aux modèles locaux.

déplacements ou en efforts ou utiliser d'autres méthodes plus évoluées?

La deuxième tient au caractère purement descendant de la démarche, les modifications apportées étant validées si elles permettent de satisfaire aux critères réglementaires, ce qui peut se traduire par des conceptions «patchwork» présentant de nombreux inserts ou «patches » de matériau ou d'épaisseur différentes. On a pu montrer sur des cas réels que le dimensionnement résultant n'était pas optimal et qu'il était possible, à poids égal, de le simplifier tout en satisfaisant aux contraintes réglementaires.

Enfin, il est important de noter que la démarche réglementaire ne permet pas d'accéder simplement aux marges, les coefficients introduits dans les formules couvrant des incertitudes sur les chargements, la tenue des matériaux, les tolérances géométriques, les imperfections de fabrication, etc. Il est donc pratiquement impossible dans ces conditions de réaliser sans prendre de risques une optimisation de dimensionnement.

\subsection{Autres calculs courants}

Les calculs réglementaires sont en général complétés par d'autres études dont les plus importantes sont les études dynamiques. En effet les navires voient de nombreuses sollicitations dynamiques, en particulier celles liées à la propulsion. Il est donc important d'en analyser le comportement vibratoire, en particulier dans la bande $0,7-80 \mathrm{~Hz}$. Les basses fréquences correspondent aux modes de poutre navire qui peuvent être excités par la houle, à certaines fréquences de rencontre, ce qui provoque des phénomènes résonant dits de «springing », ou par des impacts fluides (tossage ou slamming) induisant des réponses transitoires de fouettement ou whipping (Ledoux et al. [5]). On rencontre ensuite à partir de $7 \mathrm{~Hz}$ par exemple, des fréquences de sous-ensembles comme les superstructures, mâts radars, d'appendices comme les chaises de ligne d'arbres ou safrans, de ponts qui peuvent entrer en résonance avec les fluctuations de pression et de poussée générées par la propulsion. Il existe des classes de confort éditées par les sociétés de classification et une norme iso spécifiant des niveaux vibratoires à ne pas dépasser dans les zones publiques (équipages et passagers). De plus, il importe de vérifier, hors zones publiques, que les niveaux vibratoires ne risquent pas de nuire à terme à l'intégrité de la structure en provoquant des ruptures en fatigue ou à des dysfonctionnements graves des équipements. Ces études s'appuient en général sur le modèle éléments finis global réalisé pour les études statiques et comportent une analyse modale et une réponse fréquentielle, les excitations étant pour l'essentiel harmoniques (fluctuations de poussée et de pression dues à la propulsion, excitations moteurs). Sur les grands navires à passagers, la densité modale est très élevée et comporte de nombreux modes locaux tels les modes de mailles de ponts entre raidisseurs. Afin de filtrer ces modes, on réalise en général une réduction de modèle par condensation de Guyan. On calcule néanmoins de l'ordre de 600 modes dans la bande $[0,30 \mathrm{~Hz}]$ à partir d'un modèle comportant 300000 degrés de liberté.

Une deuxième difficulté liée aux études dynamiques tient aux incertitudes liées à la distribution de masse. Pour un navire à passagers, la masse de la structure résistante ne représente qu'un tiers de la masse totale, les gros équipements et capacités un autre tiers, la masse restante venant des équipements et revêtements divers dont la localisation n'est connue au mieux que par zones. Les modèles d'amortissement généralement utilisés sont des amortissements modaux qui représentent mal l'hétérogénéité de comportement liée en particulier aux différents types de revêtements de ponts (matériaux viscoélastiques, bétons légers, etc.), et l'amortissement apparent lié à l'énergie vibratoire absorbée par la forte densité d'équipements attachés à la structure. Des méthodes de prise en compte d'amortissement régional ont été développées mais butent sur la rareté des mesures permettant de les alimenter. Notons enfin que ces calculs dynamiques prennent en compte le couplage fluide structure (Fig. 4), que le fluide soit interne (cas des LNG par exemple) ou externe et semi-infini, ce qui se traduit par des matrices de masse ajoutée. Ces matrices 


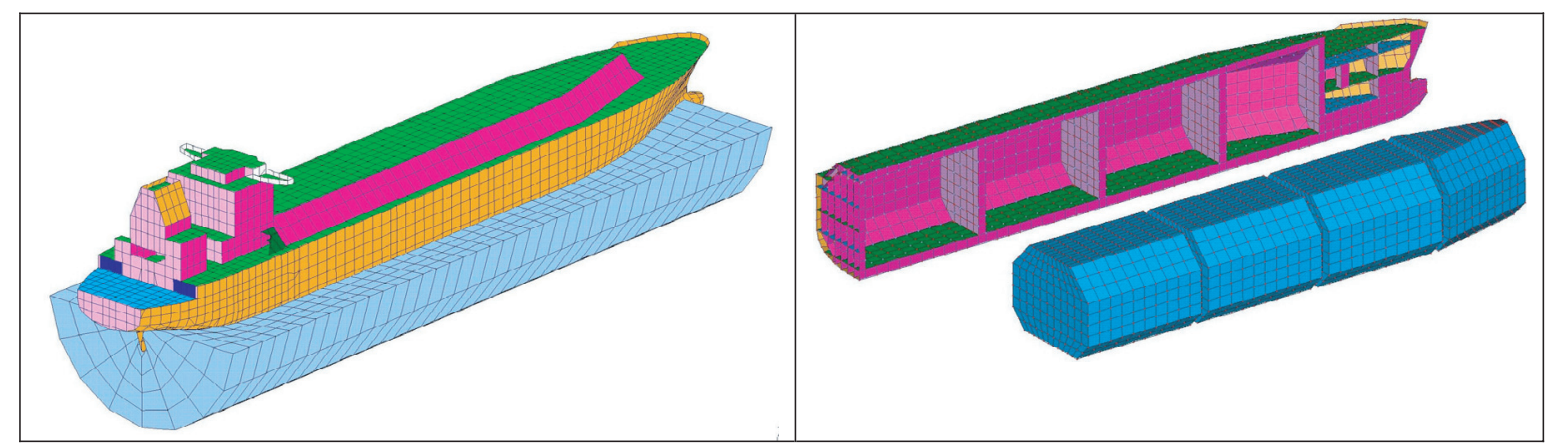

Fig. 4. Couplage fluide structure, modèles fluides internes et externes.

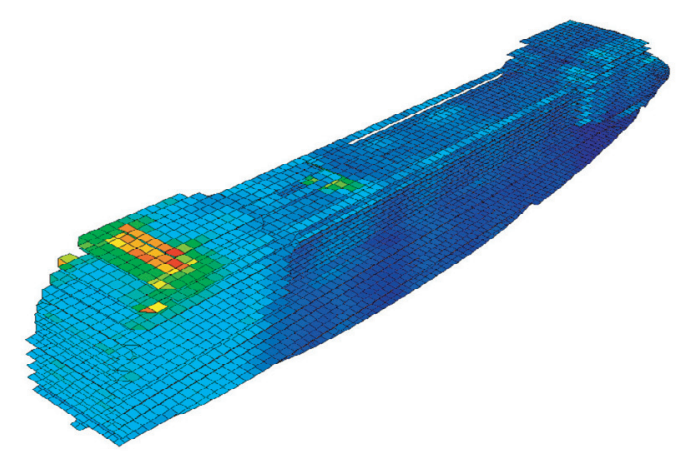

Fig. 5. Réponse fréquentielle, cartographie des vitesses.

sont calculées par éléments finis/semi-infinis fluides ou équations intégrales (Besnier et al. [6], Besnier et Jian [7]).

La figure 5 montre un exemple de réponse harmonique représentée par la cartographie des vitesses verticales maximales dans la bande de fréquence étudiée.

\section{4 Évolutions et tendances actuelles}

\subsection{Une meilleure prise en compte des chargements}

Les progrès des logiciels de simulation et l'accroissement des performances des ordinateurs permettent d'estimer les chargements vus par un navire considéré comme un corps rigide. Des logiciels comme Diodore (Ledoux et al. [5]), développé à Principia, reposant sur une théorie potentiel, permettent de calculer les distributions de pression sur une carène 3D dans le domaine fréquentiel (amplitude et phase) ou temporel, pour différentes caractéristiques de houle et angles d'incidence du navire par rapport à celle-ci. La vitesse d'avance peut être prise en compte de manière approchée. Certes, tous les effets nonlinéaires dus aux formes avant et arrière des navires et à la complexité d'une houle réelle ne peuvent pas être actuellement pris en compte. Les développements en cours dans les codes de CFD permettent d'envisager des progrès sensibles, mais les calculs restent encore trop lourds pour pouvoir être utilisés pour des études industrielles d'ensemble (De Jouëtte et al. [8]).
La prédiction des chargements impulsifs dus aux impacts de fluides, qu'ils proviennent du ballottement dans les citernes ou du tossage (impacts carène surface libre) donne lieu à de nombreux travaux théoriques et expérimentaux. On sait à peu près estimer la résultante de ces chargements impulsifs, ce qui est suffisant pour simuler la réponse d'ensemble du navire. Mais l'analyse des phénomènes locaux, pour lesquels il est nécessaire de prendre en compte l'effet des déformations de la zone impactée sur l'écoulement pose encore un certain nombre de difficultés et fait l'objet de recherches nombreuses (Couty et al. [9]). Différentes méthodes sont utilisées comme les méthodes éléments finis en formulation ALE, ou couplage Euler Lagrange, les volumes finis, avec suivi de la surface libre par méthode VOF, les méthodes SPH, etc. (Fig. 6).

Par ailleurs, les progrès réalisés dans l'observation des océans permettent de disposer de données statistiques sur les états de mer, données d'entrée des calculs en fatigue. On a donc accès à des modèles de chargement réalistes permettant une approche rationnelle du comportement des navires.

\subsection{Fatigue et optimisation}

Un des problèmes majeurs posé par la conception des structures de navires est celui de la fatigue, compte tenu de leur durée d'exploitation, de la complexité et de la nature stochastique des chargements, et des incertitudes sur les conditions d'exploitation du navire.

L'approche réglementaire spécifie des valeurs de contraintes à respecter, valeurs couvrant la fatigue. La difficulté consiste alors à calculer les contraintes locales significatives. On sait que les fissures vont s'amorcer au voisinage des cordons de soudure, dans des zones fortement sollicitées. La finesse des maillages des modèles d'ensemble ne permet pas de calculer avec une précision suffisante ces contraintes. On réalise donc des modèles locaux fins de détails identifiés et connus par expérience pour être sensibles à la fatigue (Fig. 7). Ces modèles détaillés sont ensuite chargés par des sollicitations simples (traction compression), tirées des résultats de l'analyse globale. Les valeurs de contraintes sont ensuite entrées dans des courbes SN disponibles pour des détails types, ce qui permet de connaître le nombre de cycles admissibles (Det Norske Veritas [10]). Rappelons que les courbes 


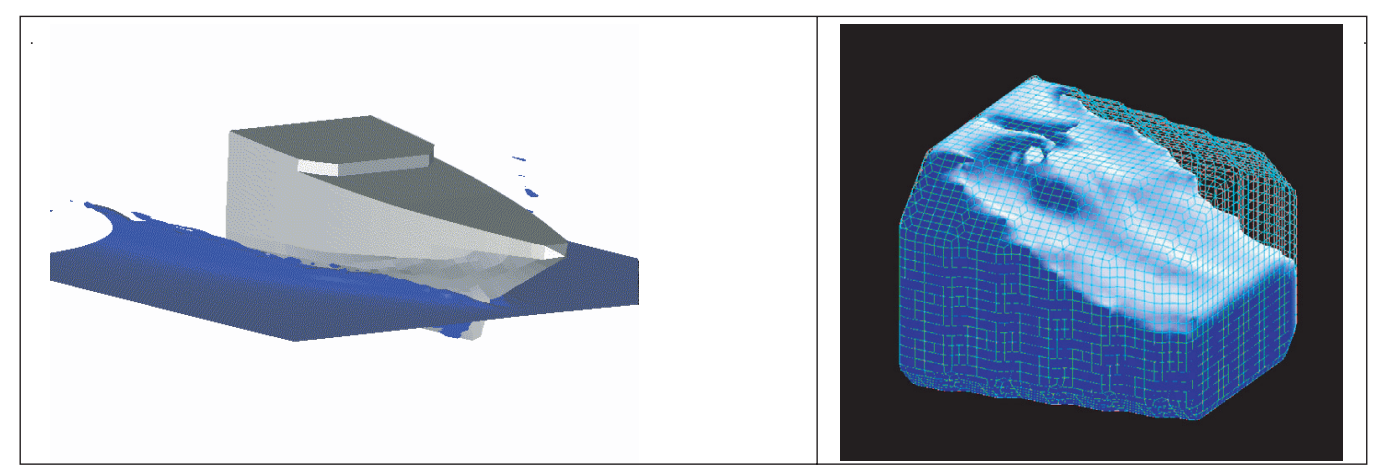

Fig. 6. Simulation de slamming (Euler Lagrange) et de sloshing (Vol. Finis, VOF).

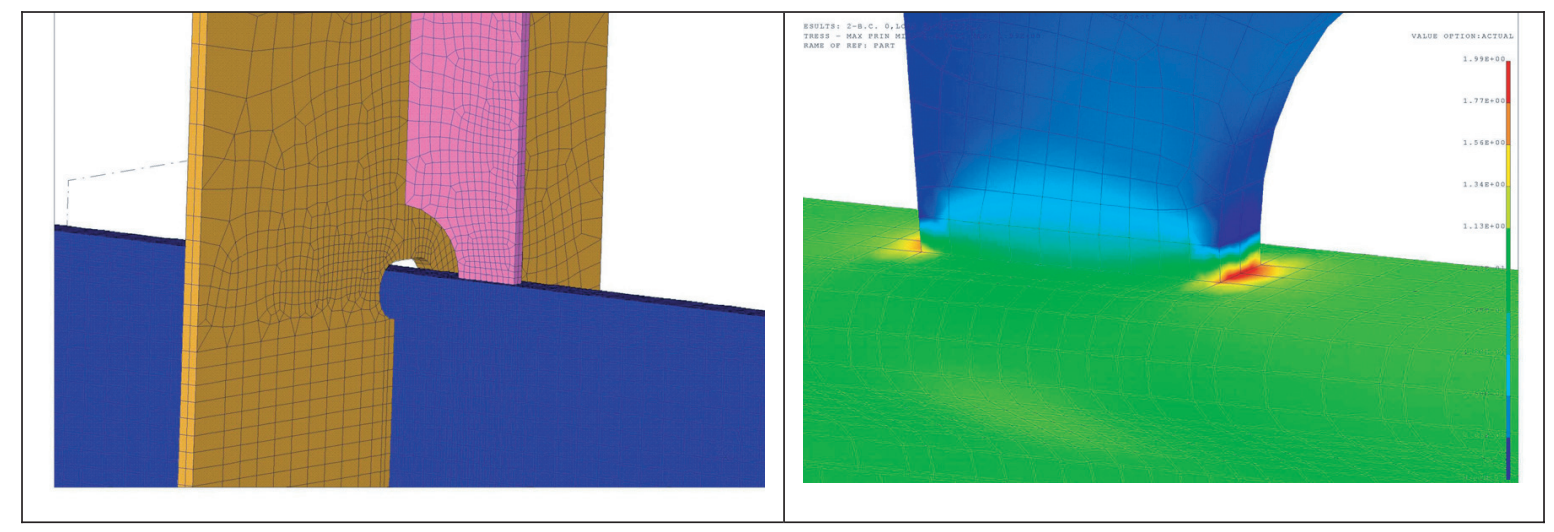

Fig. 7. Modèle local pour une étude de fatigue.

SN donnent pour une éprouvette ou un assemblage donné, le nombre de cycles conduisant à la rupture en fonction de l'amplitude de variation de contrainte.

Ces approches tendent à être remplacées par des méthodes de calcul plus explicites, comme l'approche spectrale. Dans celle-ci, on calcule dans le domaine fréquentiel, donc à partir d'un modèle éléments finis dans une approche dynamique, les fonctions de transfert (Response Amplitude Operators : RAOs) de réponse du navire, pour différentes houles unitaires régulières de caractéristiques différentes (périodes, hauteurs significatives), plusieurs angles d'incidence et fréquences de rencontre, les chargements correspondant en terme de distribution de pression étant obtenues par des codes de tenue à la mer 3D. Un état de mer court terme $H_{\mathrm{s}}$, tel que l'état de mer ne change pas pendant une certaine durée, peut être caractérisé par un spectre $S_{\mathrm{w}}(\omega)$ représentant la distribution de l'énergie de la houle en fonction de la fréquence. La réponse du navire court terme est alors obtenue comme suit : $S_{\mathrm{M}}\left(\omega_{\mathrm{e}}\right)=\left|\Phi\left(\omega_{\mathrm{e}}\right)\right|^{2} S_{\mathrm{w}}\left(\omega_{\mathrm{e}}\right), \Phi\left(\omega_{\mathrm{e}}\right)$ étant la fonction de transfert correspondant à une fréquence de rencontre $\Phi\left(\omega_{\mathrm{e}}\right)$. On calcule ensuite le dommage long terme, compte tenu de la probabilité de rencontrer un état de mer à vitesse et cap donné pendant la vie du navire (Fig. 8).

Cette approche repose sur des hypothèses de linéarité que l'on sait simplificatrices, les efforts dus à la houle étant des fonctions non-linéaires du tirant d'eau instantané, lequel dépend à son tour des mouvements de la mer et du navire. De plus, elle ne permet pas de prendre en compte les effets du slamming, décrits par une réponse temporelle. Enfin, elle suppose que la réponse est un processus à bande étroite, hypothèse trop simplificatrice dans certains cas. On est donc amené à réaliser des approches purement temporelles de comportement du navire permettant de cumuler différentes sollicitations. Ces historiques de réponse temporelle peuvent être déduits par des techniques de convolution appliqués aux résultats de réponse fréquentielle et/ou par intégration directe des équations du mouvement. Une méthode de comptage du type « rainflow » conduit ensuite aux durées de vie. Cette durée de vie est bien entendu contrôlée à chargement donné par le niveau de contrainte local. Les méthodes d'optimisation de forme, topologie et dimensionnement, disponibles maintenant dans de nombreux logiciels, permettent d'aboutir à des dessins de détail améliorant très sensiblement les durées de vie.

\subsection{Acoustique}

La prédiction du niveau de bruit dans les navires donne lieu à de nombreux travaux et si pour l'essentiel, elle repose sur des méthodes semi-empiriques associant mesures et calculs, deux classes de méthodes tendent à émerger : les méthodes Statistic Energy Analysis (SEA) et plus récemment des méthodes éléments finis énergétiques (EFA) partant de la similitude entre les équations de 

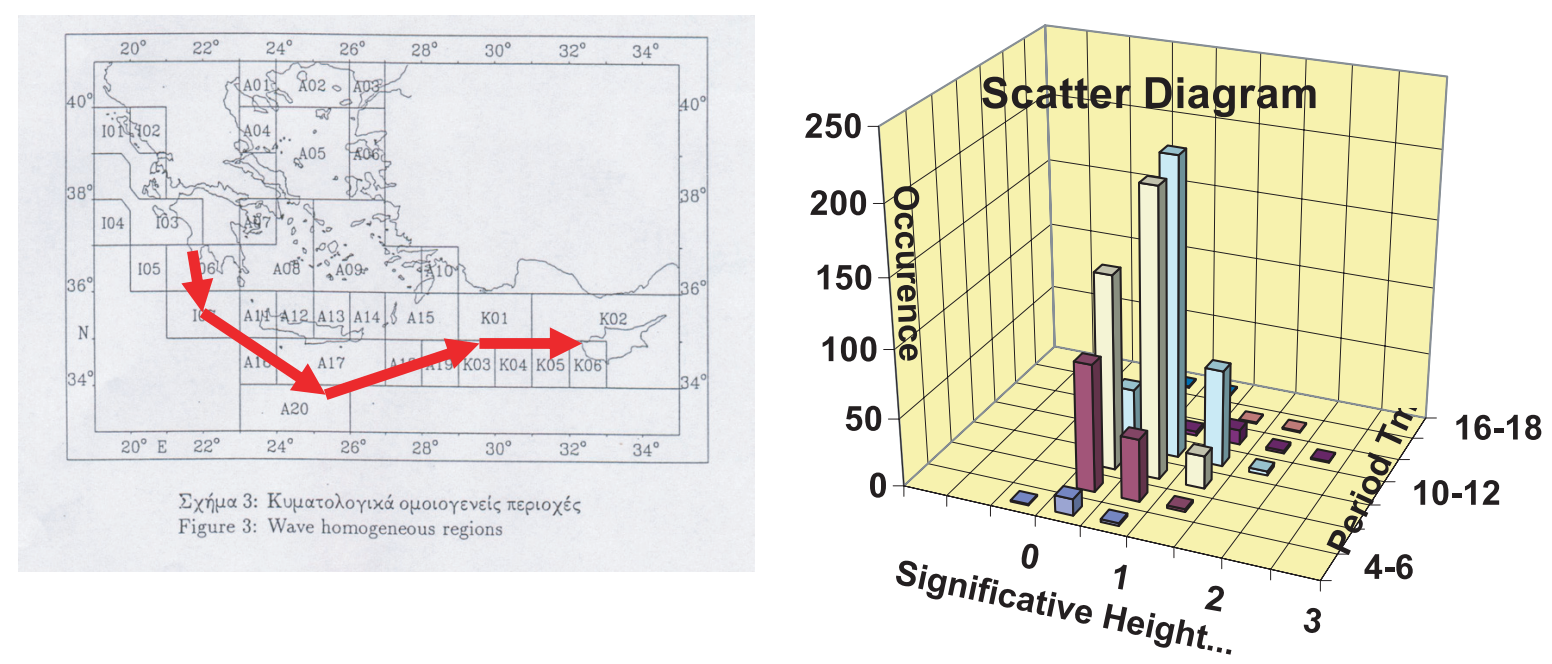

Fig. 8. Exemple de route et distribution d'états de mer.
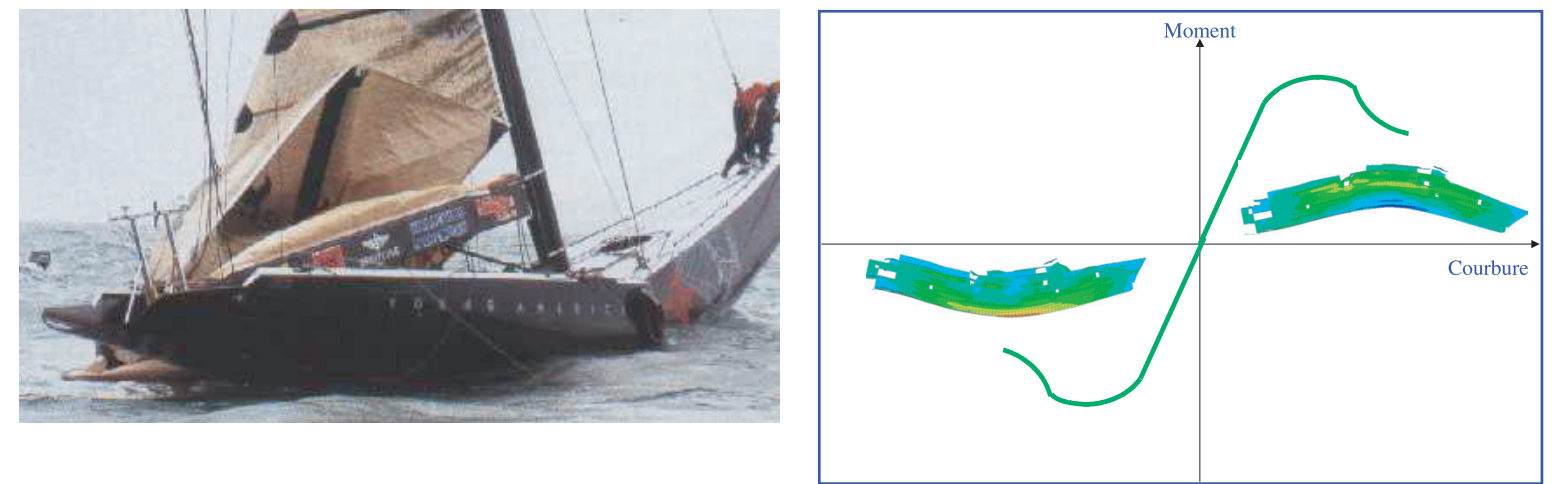

Fig. 9. Rupture par dépassement de la résistance ultime et courbe moment courbure en arc et contre-arc.

propagation de la chaleur et de propagation de l'énergie acoustique (Bernhard [11], Cabos [12]). S'il existe des logiciels SEA commercialisés, ça n'est pas encore le cas des logiciels EFA.

Le problème du bruit rayonné dans l'eau soulève encore pas mal de difficultés liées au temps de calcul et à la précision. Les approches éléments finis, semi-infinis et équations intégrales sont utilisées, comme par exemple dans Turkel et al. [13].

\subsection{Situations extrêmes et accidentelles}

\subsubsection{Résistance ultime}

La ruine des navires peut être due à des phénomènes de fatigue et à la propagation de fissures associée. Mais elle peut être aussi provoquée par des phénomènes de flambement de panneaux raidis et de plastification. Le module d'inertie de la poutre navire décroît et les phénomènes de flambement et de plastification peuvent se propager jusqu'à la ruine de la structure.

Il est donc important de connaître quelle est la résistance ultime, autrement dit le moment fléchissant maximum que peut rencontrer un navire sans se casser
(Fig. 9). On s'intéresse en général au maître couple, qui apparaît comme déterminant. Il est possible de réaliser un calcul incrémental non-linéaire à partir d'un modèle éléments finis 3D, mais de tels calculs sont longs à mettre en ouvre et à réaliser et ne se prêtent pas à des études paramétriques. Des approches plus ou moins complexes de la résistance ultime de poutre navire ont été et sont développées en discrétisant la coupe au maître par des composants élémentaires dont les lois de comportement peuvent être complexes, sans couplage de ces éléments (Pradillon et al. [14], Paik et al. [15]) ou avec couplage (méthodes ISUM). Ces méthodes peuvent être intégrées dans une approche probabiliste prenant en compte les dispersions de comportement et les approximations du réel (Jancart et al. [16]).

\subsubsection{Collisions}

Avec l'accroissement du trafic maritime, les risques de collisions augmentent considérablement en particulier dans les détroits et au voisinage des grands ports. Ces collisions peuvent être à l'origine de pollutions importantes et des travaux de recherche visent en particulier à minimiser les conséquences de collisions mineures en prévoyant 


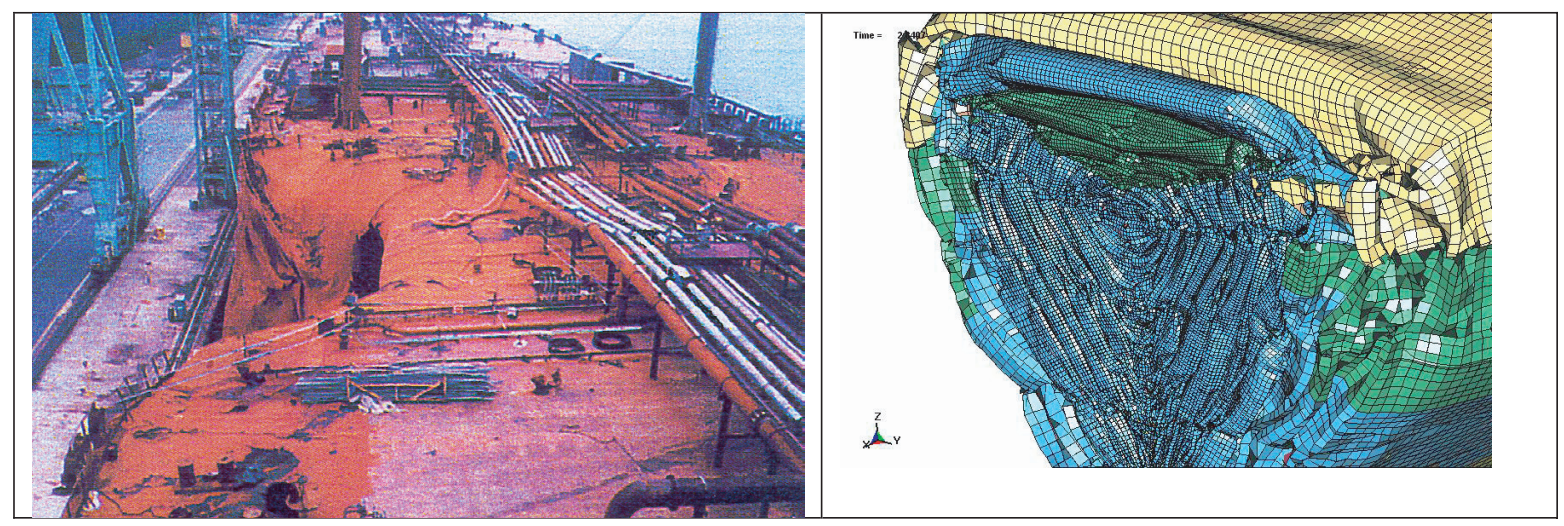

Fig. 10. Exemple de collision réelle et de simulation.

à la conception des structures capables d'absorber une partie de l'énergie cinétique. Il est évident que les essais à l'échelle 1 sont pratiquement exclus et la simulation numérique joue dans ce domaine un rôle particulièrement important. Mais l'étude de ces collisions, qui s'apparente à des études de crash telles que celles effectuées pour les transports terrestres, présente un certain nombre de difficultés supplémentaires. La collision comporte un choc localisé suivi du mouvement du navire impacté, ces deux phénomènes ayant des durées très différentes. De plus, il s'agit d'un problème couplé, une partie de l'énergie étant dissipée dans les mouvements du fluide. Ces études sont réalisées par exemple avec des codes de dynamique rapide explicites comme LS-DYNA couplés à un module de mouvements de corps rigides en présence de fluide (Donner et al. [17]) (Fig. 10)

\subsubsection{Explosions}

Les explosions sous-marines ou aériennes concernent essentiellement les navires militaires mais certaines études de ce type peuvent aussi intéresser les navires civils comme les navires de prospection sismique et les unités de production et de stockage pétrolier offshore (FPSO). De telles études ont pour but de vérifier la tenue locale de la structure mais aussi de connaître la réponse d'ensemble du navire (fouettement), en particulier les niveaux d'accélération que peuvent voir certains équipements. Il s'agit, comme dans le cas de collisions, de phénomènes couplés fluide structure multi-échelles en temps et espace. Ils sont étudiés à l'aide de codes explicites couplés à des formulations équations intégrales ou éléments finis acoustiques, une des difficultés venant de la taille des modèles fluides.

\subsection{Approches fiabilistes}

À l'exception des chargements, l'étude du comportement des navires est réalisée de façon déterministe. La prise en compte des incertitudes, dans des approches fiabilistes fait l'objet de travaux et de développements devant déboucher à terme sur des représentations plus fines de la réalité. Dans ces approches, on recherche la probabilité d'atteindre et de dépasser un état limite, compte tenu des incertitudes et de la nature stochastique de données du modèle. On a vu le caractère clairement stochastique des chargements. Mais il est possible de définir d'autres variables stochastiques permettant de prendre en compte des méconnaissances, dispersions et incertitudes : dispersion des propriétés de matériaux, en particulier la contrainte limite, des épaisseurs de tôles, des courbes $\mathrm{SN}$, incertitudes liées aux approximations inhérentes à la modélisation, non prise en compte des déformations et contraintes générées au cours de la fabrication, incertitudes sur la répartition des masses, etc. Les méthodes fiabilistes ne sont pas utilisées pour des analyses globales mais portent sur des études partielles comme la résistance ultime et la fatigue de détails. Elles sont utilisées par les Sociétés de classification pour calibrer les facteurs de sécurité. Leur utilisation est amenée à se développer à l'avenir (Jancart et al. [16], Maherault et al. [18]).

\section{Conclusions}

La conception des structures de navires, dans un cadre règlementaire lié à la sécurité, de concurrence très vive et dans un contexte industriel particulier (délais très courts et pas de série) fait largement appel à la simulation numérique. Le domaine le plus complexe, qui nécessite des outils spécifiques concerne les chargements. Les travaux en cours cherchent à mieux appréhender la réalité en prenant en compte les non-linéarités et par une meilleure modélisation des phénomènes stochastiques. On dispose pour les calculs de structures de codes éléments finis généralistes, qui peuvent, complétés par des post-processeurs métier spécifiques, être utilisés sans ajouts majeurs pour la plupart des calculs courants. Les problèmes posés résident essentiellement dans l'analyse de phénomènes couplés fluide structure, qui peuvent se traiter par couplage de codes et, d'une façon générale dans l'intégration de différents modules dans une chaîne de calcul cohérente. En outre, les problèmes de modélisation et 
de stratégie d'étude se posent, compte tenu en particulier de la taille des structures. Le développement et l'utilisation de méthodes multi-échelles apporteraient un progrès important, en particulier pour faciliter et améliorer la précision des études locales. Les calculs non-linéaires : impacts, collisions, explosions, bien que possibles restent encore délicats et demandent une bonne expertise. Ils sont souvent utilisés pour valider et calibrer des méthodes plus simples et plus rapides à mettre en œuvre, compatibles avec les délais d'étude extrêmement courts. Mentionnons enfin le développement des approches fiabilistes, permettant de mieux maîtriser les marges et donc d'optimiser la conception en contrôlant les risques. Comme dans d'autres secteurs industriels, le concept de maquette numérique fait son chemin, mais s'applique actuellement plus aux problèmes d'emménagements et à la production qu'à la simulation numérique.

\section{Illustrations}

Les figures 2 à 5 proviennent de modèles réalisés pour les Chantiers de l'Atlantique.

\section{Références}

[1] J.C. Masson, Évaluation par le calcul du comportement mécanique des structures navales, Proc. du $3^{\mathrm{e}}$ colloque en calcul des structures, Giens, Hermès, 1997

[2] Bureau Veritas Rule for Steel ships (2000)

[3] T.T. Chau, F. Besnier, Numerical simulation of welding in shipbuilding, 5th Int. Conf. On Marine Technology and transportation, Szcezecin, Poland, 2003

[4] M. Gérardin, D. Rixen, Théorie des vibrations, Masson, 1996

[5] A. Ledoux, C. Mary, N. Couty, Modeling of springing and whipping of FPSOs in a time domain sea-keeping tool, Proc. ISOPE congress, Toulon, 2004

[6] F. Besnier, G. Bechepay, Y. Mavrakakis, M. Ferry, Vibration Analysis Method of Ship Structures in the Medium Frequency Domain, Proc. 8th Int. Symp. PRADS 2001, Wu Y., Cui W. et Zhou G. (Eds.), Elsevier, Shangaï, China, Elsevier (2001) 1177-1184
[7] F. Besnier, L. Jian, Fluid Structure Coupled Analyses in Shipbuilding Using Permas FS, Finite Element in Engineering Applications INTES GmbH, Heidelberg, Germany, 2002

[8] C. De Jouëtte, O. Laget, J.M. Le Gouez, Vers la tenue à la mer par une formulation en fluide visqueux, Septièmes journées de l'hydrodynamique, Marseille, 1999

[9] N. Couty, L. Jian, Tree dimensional simulation of slamming and whipping responses of a fast ship with finite element software, NAV2003 proceeding, Palermo, 2003

[10] Det Norske Veritas, Fatigue assessment of ship structures, Classification notes $\mathrm{N}^{\circ} 30.7,2001$

[11] R.J. Bernhard, The family of EFA equations and their relationship to SEA, Proc. Int. Conf. Noise and Vibration Energy Methods, November 2000, France

[12] C. Cabos, H. Matthies, A method for the prediction of structure-borne noise propagation in ships, Proc. of the 6th International Congress on Sound and Vibration, Technical University of Denmark, 1999

[13] E. Tukel, C. Farhat, U. Hetmaniuk, Improved Accuracy for Helmholz Equation in unbounded domains, Int. J. Numer. Meth. Engn, 59 (2004) 1963-1988

[14] J.Y. Pradillon, T. Quesnel, C. Toderan, P. Rigo, Ultimate strength of longitudinally stiffened panels : multicriteria comparative analysis, Proc. 8th Int. Symp. PRADS 2001, Wu Y., Cui W. et Zhou G. (Ed.), Elsevier, Shanghai, China, Elsevier, 2001, pp. 1029-1035

[15] J.K. Paik, A.K. Thayamballi, Ultimate strength of aging ships, J. Engineering for the maritime environment, 216 (2002) $57-77$

[16] F. Jancart, F. Besnier, Reliability Analysis of ship structures fatigue and ultimate strength, ASRANET Int. Coll., University of Glasgow, 2002

[17] R. Donner, F. Besnier, H. Le Sourne, Numerical Simulation of Ship-Submarine Collisions, Proc. 8th Int. Symp. PRADS 2001, Wu Y., Cui W., Zhou G. (Eds.), Elsevier, Shangaï, China, Elsevier, 2001, pp. 1309-1314

[18] S. Maherault, M. Huther, G. Parmentier, N. Recho, Semi probabilistic Fatigue calibration of the partial safety factor, IIW-Doc XIII, 1794-1799, Ins. Institute of welding, 1999

[19] R.H. Lyon, G. DeJong, Theory and Aplications of Statistical Energy Analysis, Butterworth Heineman, Boston, 1985

[20] Y. Ueda, S.M.H. Rashed, ISUM (Idealized Structural Unit Method) Applied to Marine Structures. Trans. JWRI 20 (1991) 123-136

$\overline{\text { Retrouvez nos articles sur le site : }}$ www.edpsciences.org/meca 